Orthopäde 2010 · 39:236-236

DOI 10.1007/s00132-009-1540-5

Online publiziert: 24. Februar 2010

(c) Springer-Verlag 2010

\author{
M.A. Rauschmann ${ }^{1} \cdot$ K.D. Thomann ${ }^{2}$ \\ ${ }^{1}$ Abteilung für Wirbelsäulenorthopädie, Orthopädische Universitätsklinik \\ Friedrichsheim gGmbH, Frankfurt/Main \\ ${ }^{2}$ Institut für Gutachtenmedizin, Frankfurt/Main
}

\title{
Wirbelsäulenverletzungen aus gutachterlicher Sicht
}

und Empfindungsstörungen, die aus gutachterlicher Sicht auf dem Boden des orthopädisch/unfallchirurgischen Fachgebiets keine Basis haben. Dieser Problematik soll dieses Themenheft Rechnung tragen. Es ist eine Zusammenstellung einer gutachterlichen Tagung des vergangenen Jahres, welche von den Unterzeichnern geleitet wurde.

Das vorliegende Themenheft soll dem gutachterlich tätigen Kollegen als Basis für die Erstellung von Gutachten zu dieser Thematik dienen. Ein großer Wert wurde auf die jeweiligen Literaturrecherchen $\mathrm{zu}$ den Teilgebieten gelegt, die dem Leser als Pool für zukünftige Gutachtenerstellungen dienen können. Es wurden bewusst zur Einführung Übersichtsartikel zu Wirbelsäulenverletzungen der Hals- sowie der Brust- und Lendenwirbelsäule ausgewählt, die die Pathogenese, Biomechanik, Diagnostik und Therapie zur Darstellung bringen. Den Folgen einer Verletzung im Sinne der posttraumatischen Kyphose im thorakolumbalen Übergangsbereich wurde mit einem gesonderten Beitrag Rechnung getragen. Im Weiteren wird der Unfallananlyse, der Untersuchungstechnik aus gutachterlicher Sicht bei Wirbelsäulenverletzungen, wie auch dem Schwerpunktthema der HWS-Schleuderverletzung sowie der psychischen Begleitkomponenten solcher Verletzungen ausreichend Platz eingeräumt.

Dieses Heft soll somit ein "Update“ aus interdisziplinärer Sicht sein und bei zukünftigen Problemfällen als aktuelles Nachschlagewerk dienen.

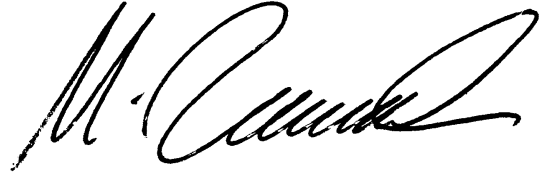

M.A. Rauschmann

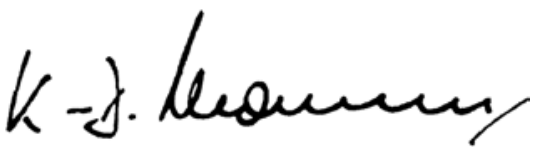

K.D. Thomann

\section{Korrespondenzadresse \\ PD Dr. M.A. Rauschmann}

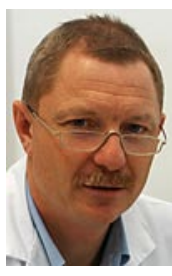

Abteilung für Wirbelsäulenorthopädie, Orthopädische Universitätsklinik Friedrichsheim gGmbH Marienburgstraße 2 60528 Frankfurt/Main m.rauschmann@friedrichsheim.de 